\title{
A Novel Magnetic Rotary Encoder for Servo Motors
}

\author{
Takefumi Kabashima Member (Yaskawa Electric Corpolation, kabashima-ad@adp.jiii.or.jp) \\ Yuji Arinaga Non-member (Yaskawa Electric Corpolation, arinaga@yaskawa.co.jp) \\ Koji Uemura Non-member (Yaskawa Electric Corpolation, ue@yaskawa.co.jp) \\ Ikuma Murokita Non-member (Yaskawa Electric Corpolation, muro@yaskawa.co.jp) \\ Motomichi Ohto Member (Yaskawa Electric Corpolation, ohto@yaskawa.co.jp)
}

Keywords: magnetic rotary encoder, FEM and servo motor

Servomotors used in semiconductor manufacturing equipment and in ultra-precise machining applications are required to be very small with fast and precise controllability. The sensors for detecting rotational speed and position hence need to be very small and have high resolution. In many applications, the absolute type sensors are preferred over the incremental type. Rotary encoders are mainly classified as optical and magnetic types that use magneto-resistive (MR) devices. Optical encoders comprise of light emitting diodes (LED), photo diodes (PD) and a rotary disc with slits. Slits are precisely formed using semiconductor process technology. Hence, in general, optical encoders have high resolution. However, in smaller than $10 \mathrm{~mm}$ size, the resolution becomes unfavorable due to the limitations imposed by the slit width and the size of LED and PD.

A new magnetic rotary encoder is proposed that addresses these problems. Fig. 1. shows a perspective view of the proposed magnetic rotary encoder for detecting the absolute position of a rotating body. It consists of a disc magnet magnetized in one direction with all its magnetic domain aligned parallel to each other, hall effect devices in the air gap, and a fixed body formed by a ring-shaped magnetically soft material.
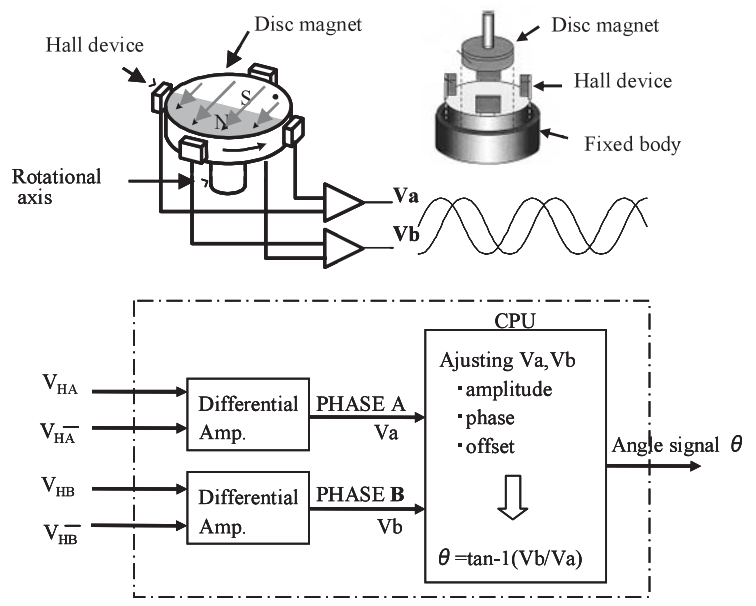

Fig. 1. Structure and processing unit of the magnetic encoder
The accuracy of the encoder depends on the distortion of pseudosinusoidal output waveform from the hall sensors. Therefore, for achieving high accuracy the distortion of the output waveform should be minimal. To obtain an encoder with high accuracy, a study of the parameters that influence the shape and material of an encoder head was carred out using FEM analysis.

An encoder based on the proposed method having a width of $10 \mathrm{~mm}$, a height of $10 \mathrm{~mm}$, and a depth of $3 \mathrm{~mm}$ was developed. The photograph of a disassembled rotary magnetic encoder is shown in Fig. 2. The output waveforms of phase A and B are shown in Fig.3. Significantly improved sinusoidal waveforms with distortion levels less than $0.6 \%$ of the fundamental was achieved. The prototype encoder has a high resolution of 4,096 ppr and is capable of detecting absolute angular position. The construction is very simple, resulting in a highly reliable encoder from vibration and operating temperature points of view.

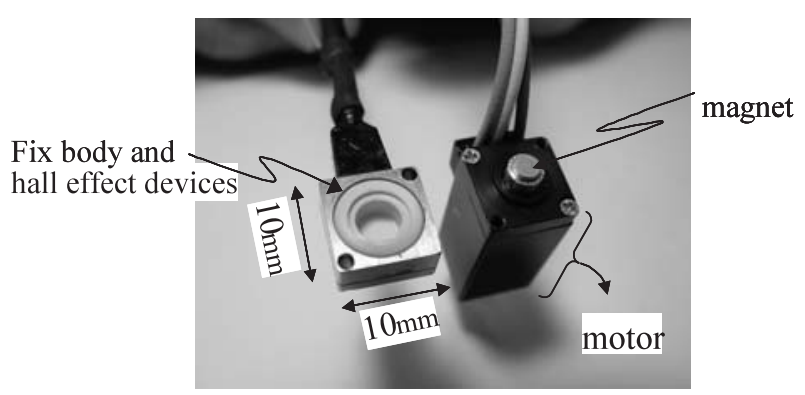

Fig. 2. Prototype of magnetic encoder

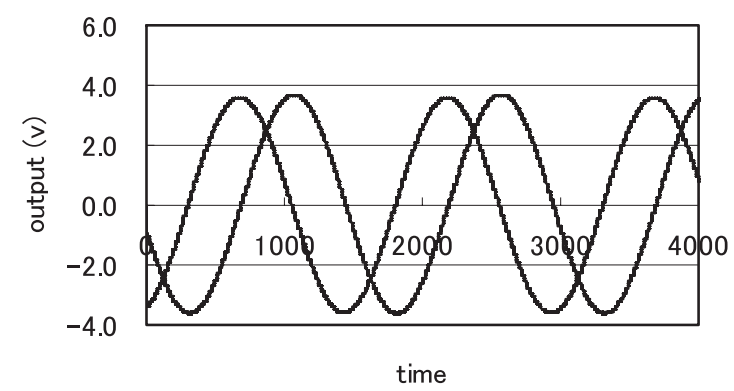

Fig. 3. Output signal of phase A and B 


\title{
A Novel Magnetic Rotary Encoder for Servo Motors
}

\author{
Takefumi Kabashima* Member \\ Yuji Arinaga* Non-member \\ Koji Uemura** $^{* *} \quad$ Non-member \\ Ikuma Murokita* Non-member \\ Motomichi Ohto* Member
}

\begin{abstract}
A new type of a magnetic rotary encoder for servomotors is proposed. A salient feature of the proposed encoder is its small size, high resolution, and simple construction. An encoder based on the proposed method having a width of $10 \mathrm{~mm}$, a height of $10 \mathrm{~mm}$, and a depth of $3 \mathrm{~mm}$ was developed and is reported here. In addition, the developed encoder has a high resolution of $4096 \mathrm{ppr}$ and is capable of detecting absolute angular position. The proposed encoder comprises of a disc magnet magnetized in one direction with all the magnetic domains in the material parallel to each other, a stationary body made up of a magnetically soft material, and four Hall effect devices. This paper describes the configuration of the encoder and the proposed detection process of the rotational position. The characteristics of the prototype encoder are also presented. The accuracy of the encoder depends on the distortion of a pseudo-sinusoidal output waveform, and therefore for achieving high accuracy the distortion of the output waveform should be minimal. The magnetic flux distribution and the output waveform of the encoder were simulated using finite element method (FEM). Based on FEM analysis, the optimal shape of the disc magnet and its housing, relative position of the Hall effect devices and the material of the disc magnet were determined to achieve high performance characteristics.
\end{abstract}

Keywords: magnetic rotary encoder, FEM and servo motor

\section{Introduction}

Servomotors used in modern semiconductor manufacturing equipment and in ultra-precise machining applications are required to be very small with fast and precise controllability ${ }^{(1)}$. The sensors for detecting rotational speed and position for such sophisticated applications are required to be very small and have high resolution. Further, in many applications, the absolute type sensors are preferred over the incremental type. Rotary encoders are mainly classified as optical and magnetic types that use magneto-resistive (MR) devices. Optical encoders comprise of light emitting diodes (LED), photo diodes (PD) and a rotary disc with slits. Slits are precisely formed using semiconductor process technology. Hence, in general, optical encoders have high resolution. However, in smaller than $10 \mathrm{~mm}$ size, the resolution becomes unfavorable due to the limitations imposed by the slit width and the size of LED and PD.

As shown in Fig. 1, magnetic encoders comprise of a magnetic drum, which is magnetized in minute pole lengths, and have MR elements. The resolution depends on the pole pitch and the spacing between the drum and the MR elements ${ }^{(2)}$. In such an arrangement, it is difficult to achieve small size and high resolution at the same time.

In this paper, a new magnetic rotary encoder is proposed that addresses these problems. It comprises of a disc magnet

\footnotetext{
* Corpolate R\&D Center, Yaskawa Electric Corporation

12-1, Ohtemachi, Kokura-kita-ku, Kitakyushu-City 803-8530

** Yaskawa Electric Corporation

480 Iruma city, Saitama Pref., 358-8555 Japan
}

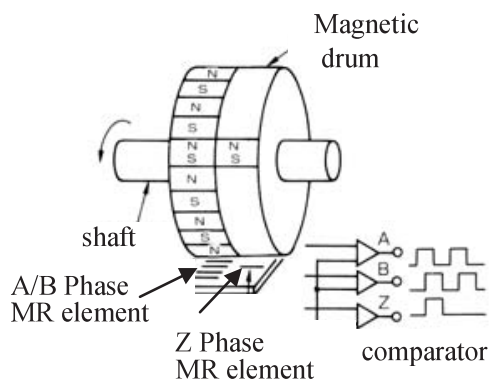

Fig. 1. Magnetic rotary encoder

magnetized in one direction with all the magnetic domains aligned parallel to each other, a fixed body formed by a magnetically soft material and Hall effect devices. The construction is very simple, resulting in a highly reliable encoder from vibration and operating temperature points of view. The accuracy of the encoder depends on the distortion of pseudo-sinusoidal output waveform from the Hall effect devices. Therefore, for achieving high accuracy the distortion of the output waveform should be minimal. The distortion of the output wave is caused by non-linearity of the magnetic property of the magnet, saturation of the stationary body that houses the Hall effect devices and the output characteristic of the Hall effect devices. The saturation of the stationary body depends on the physical dimensions of the stationary body in relation to the diameter of the magnetic disc.

The magnetic flux distributions of the encoder head were obtained using FEM taking into consideration the magnetic 
non-linearity. Next, the output waveforms were simulated, and its distortion and the position error were calculated. The optimal shape and materials of the encoder that results in high accuracy were thus determined by simulation using FEM. A prototype magnetic encoder based on the simulation result was fabricated and evaluated for its performance. A high resolution of $4096 \mathrm{ppr}$ was achieved using the fabricated encoder that was only $10-\mathrm{mmx} 10-\mathrm{mm}$ in size.

\section{Structure and Theory of Operation}

Fig. 2 shows a perspective view of the proposed magnetic rotary encoder for detecting the absolute position of a rotating body. It consists of a disc magnet magnetized in one direction with all its magnetic domain aligned parallel to each other, Hall effect devices in the air gap, and a fixed body formed by a ring-shaped magnetically soft material ${ }^{(3)(4)}$.

The uni-directionally magnetized magnet with parallel domains generates a pseudo-sinusoidal magnetic field as it rotates. The fixed body functions to increase the flux density in the air gap and to reduce magnetic flux leakage to the outside. It also prevents magnetic interference from outside sources. In the air gap, four Hall effect devices are located at regular intervals and detect the sinusoidal magnetic flux corresponding to a rotation angle. Two diametrically opposite Hall effect devices form a pair and two such pairs generate phase signals, $\mathrm{Va}$ and $\mathrm{Vb}$ that are 90 electrical degrees with respect to each other as shown in Fig. 3.
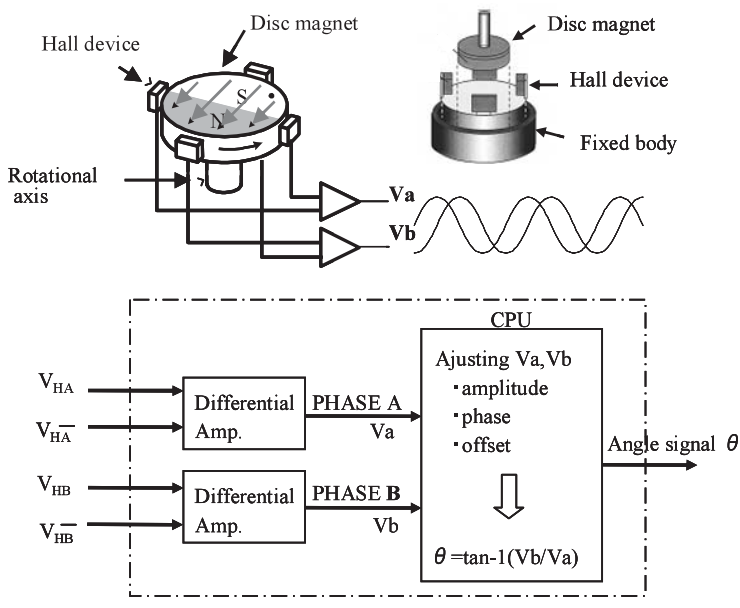

Fig. 2. Structure and Processing Unit of the Magnetic Encoder

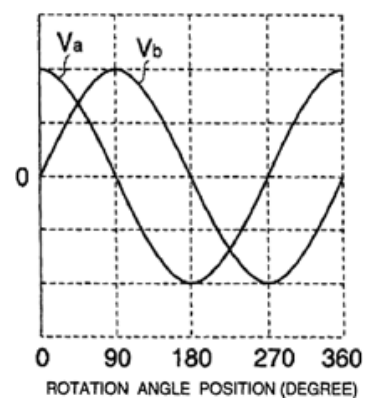

Fig. 3. Differential output signal Va, Vb
When the magnet is eccentrically rotated, the magnetic flux density in the air gap is displaced corresponding to the amount of eccentricity. However, by using differential output (Va and $\mathrm{Vb}$ ) from the two pairs of Hall effect devices, the eccentricity effect can be greatly diminished, without loss of accuracy.

The rotation angle is obtained by computing $\tan ^{-1}(\mathrm{Vb} / \mathrm{Va})$ from the differential signals $\mathrm{Va}$ and $\mathrm{Vb}$.

\section{Prospective accuracy of the encorder}

The factors that influence the error in obtaining the absolute angle of the rotor are as follows:

(1) The amplitude difference of the output signal Va and $\mathrm{Vb}$,

(2) The phase deviation from 90 degrees in electrical angle between $\mathrm{Va}$ and $\mathrm{Vb}$,

(3) The offset in $\mathrm{Va}$ and $\mathrm{Vb}$, and

(4) The distortion of the hall output waveforms.

To estimate the degree of influence of each factor affecting the accuracy, the simulations were done under the following assumptions. Va is cosine waveform and $\mathrm{Vb}$ is pseudosinusoidal waveform with second and third harmonic components.

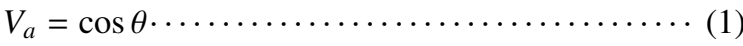

$$
\begin{aligned}
& V_{b}=(1+\alpha) \cdot\{\sin (\theta+\delta)+\beta \sin 2(\theta+\delta)+\gamma \sin 3(\theta+\delta)\}
\end{aligned}
$$

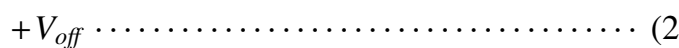

The error in the computation of the absolute angle caused by the above factors were simulated by assuming one hall sensor pair to be ideal while the other pair to have varying degrees of errors caused by the aforementioned factors. The output signals $\mathrm{Va}$ and $\mathrm{Vb}$ are thus assumed to be as follows:

$\alpha$ : the amplitude difference

$\beta$ : the amplitude of second harmonic component

$\gamma$ : the amplitude of third harmonic component

$\delta$ : the phase shift in radians

voff: offset value in volts

The error in the angle is defined by

$$
\text { err }=\theta^{\prime}-\theta
$$

where

$$
\theta^{\prime}=\tan ^{-1}\left(V_{a} / V_{b}\right)
$$

Table 1 shows the error in the simulated angle when the parameters $\alpha, \gamma, \delta$, and $V_{\text {off }}$ are varied.

The factors that contribute to the error in the angle can be eliminated by adjusting the parameters in the processing unit. However, the error due to a distorted pseudo-sinusoidal waveform cannot be eliminated. Therefore, for realizing a

Table 1. Error factors and their corresponding accuracy

\begin{tabular}{|c|lc|c|c|c|c|c|}
\hline \multicolumn{2}{|c}{ accuracy } & bit & 8 & 10 & 12 & 16 \\
\cline { 3 - 9 } & & $\mathrm{ppr}$ & 256 & 1024 & 4096 & 65536 \\
\hline \multirow{3}{*}{$\begin{array}{c}\text { angle } \\
\text { error } \\
\text { factors }\end{array}$} & diff. of amplitude & $\alpha$ & $\%$ & 5.04 & 1.23 & 0.31 & 0.02 \\
\cline { 2 - 8 } & $3 r d$ harmonic & $\gamma$ & $\%$ & 2.84 & 0.70 & 0.18 & 0.01 \\
\cline { 2 - 8 } & phase shift & $\delta$ & deg & 1.40 & 0.35 & 0.09 & 0.01 \\
\cline { 2 - 8 } & offset & $\mathrm{V}_{\text {off }}$ & $\%$ & 2.45 & 0.61 & 0.15 & 0.01 \\
\hline
\end{tabular}




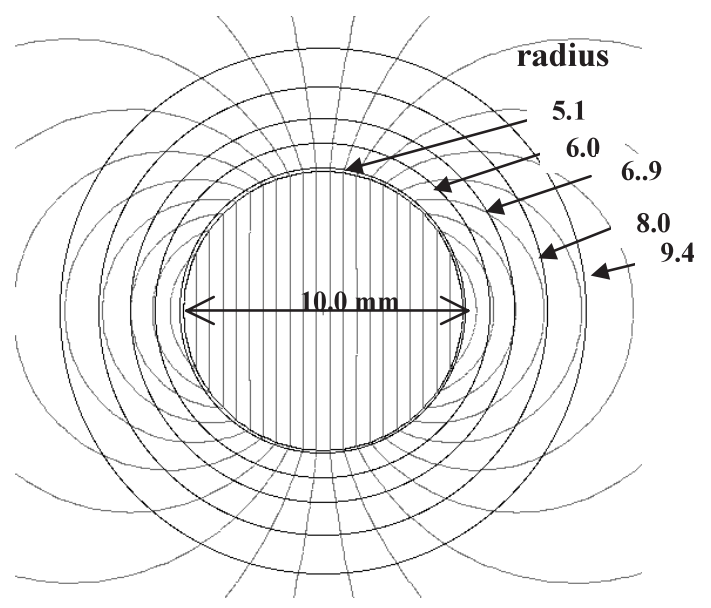

Fig. 4. The flux distribution of a disc magnet

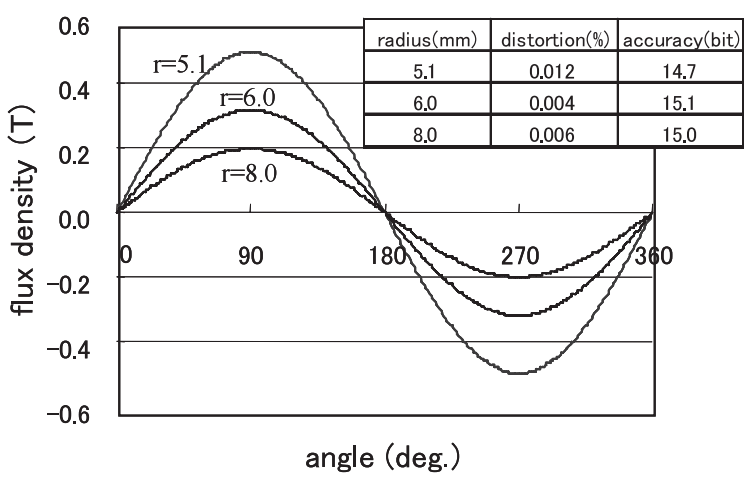

Fig. 5. The waveform of the flux density and its resulting angle computational accuracy

magnetic encoder with high accuracy, the output sinusoidal waveform from the Hall effect devices should have minimal distortion.

The distinctive feature of the new type encoder is that it has minuscule distortion of output waveform because the disc magnet used is magnetized in a uni-direction with all its magnet domains aligned parallel to each other.

Fig. 4 shows the flux distribution of the disc magnet of diameter $10 \mathrm{~mm}$. Fig. 5 shows the waveform of magnetic flux density with respect to the rotational angle. The distortions in the waveform were obtained using Fast Fourier Transform (FFT), and the error was calculated in the following way. The 90 electrical degrees phase-shifted waveforms of $\mathrm{Va}$, and $\mathrm{Vb}$ due to flux density at various radii were obtained first. The rotational angle was obtained next by computing $\tan ^{-1}(\mathrm{Vb} / \mathrm{Va})$ for each radii. The simulations indicate that the distortion of the waveforms is less than $0.01 \%$ and the accuracy is 15 bit.

\section{Optimal Design of A Magnetic Rotary En- coder for A Servomotor}

To design a magnetic rotary encoder with high accuracy, the optimal dimensions of the encoder was obtained by parameter variation study using the Finite Element Method.

4.1 An Analytical Model A magnetic rotary encoder was designed for an AC micro-servomotor. The constrains for the design are as follows:

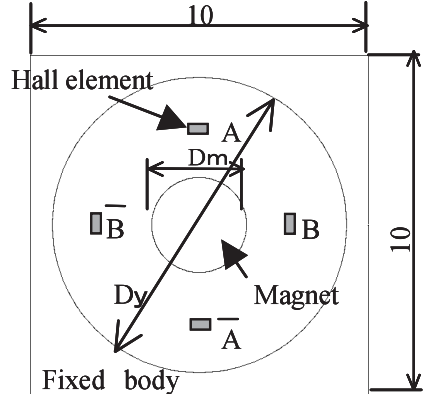

Fig. 6. Analytical model

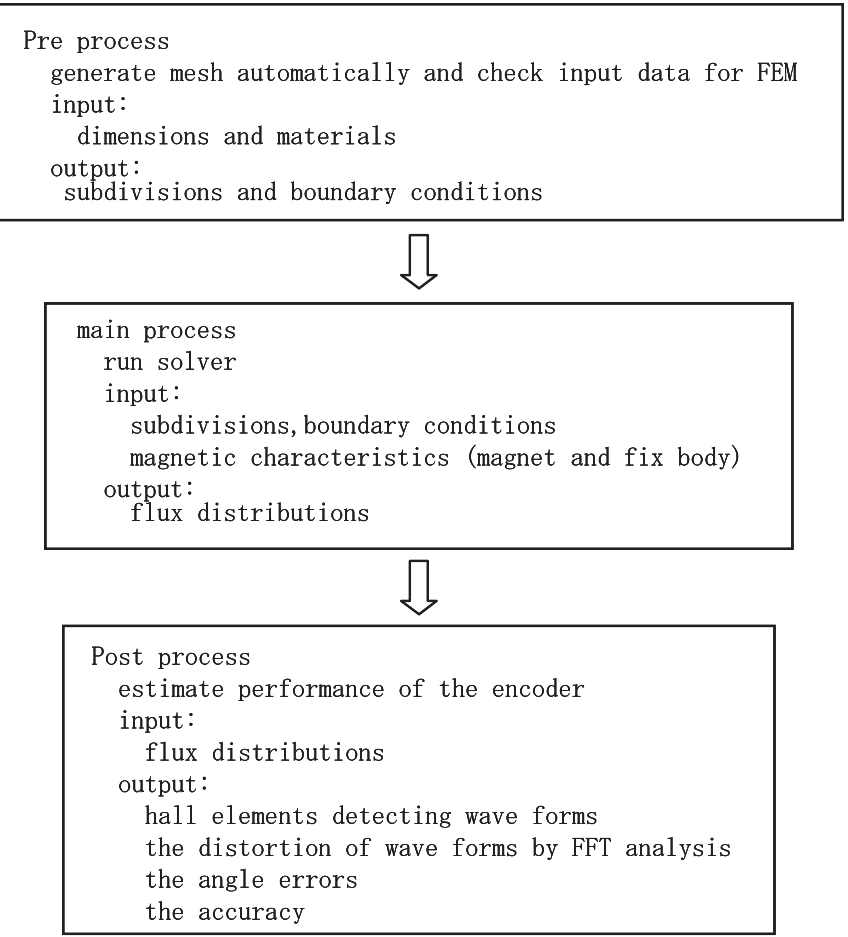

Fig. 7. Analytical flow diagram

a. The maximum outer size is $10 \mathrm{~mm}$.

b. The mechanical clearance between the rotating the magnetic disc and the fixed body of soft magnetic material is greater than $0.5 \mathrm{~mm}$.

c. Hall effect devices need a minimum radial space of $2.5 \mathrm{~mm}$.

The analytical model is shown in Fig. 6 and optimization was carried out by varying Dm and Dy using FEM. The material of the magnet and that of the fixed body were also varied to obtain the final result.

$\mathrm{NdFeB}$ magnets were chosen to increase the signal output amplitudes, and the permanent magnets have parallel anisotropy, which allows easy alignment of its entire magnetic domain in one direction parallel to each other. GaAs type Hall effect devices are chosen because of its stable temperature characteristics compared to InSb types. The stationary body that houses the Hall effect devices is made from a magnetically soft material.

4.2 Simulation process Fig. 7 shows the analytical flow diagram. When the dimensions and the materials of the 
encoder head are given, the magnetic field analysis is carried out taking into consideration the magnetic non-linearity of the magnet and the stationary body. The flux densities at

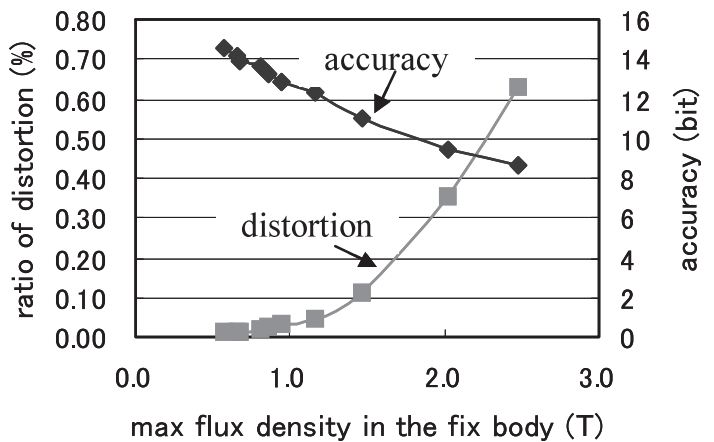

Fig. 8. Relationship between magnetic saturation, distortion, and accuracy

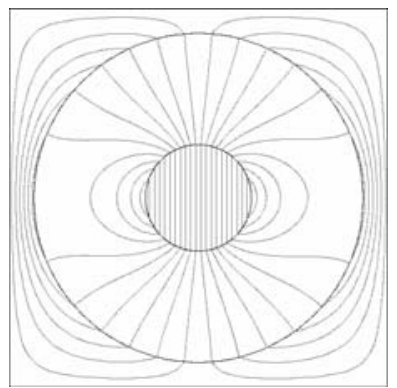

Fig. 9. Flux distribution in the optimal magnetic

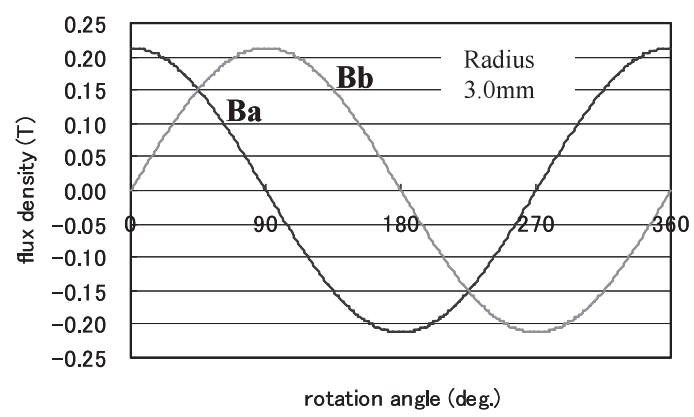

Fig. 10. Waveforms of flux density at Hall elements in the optimal structure obtained by FEM

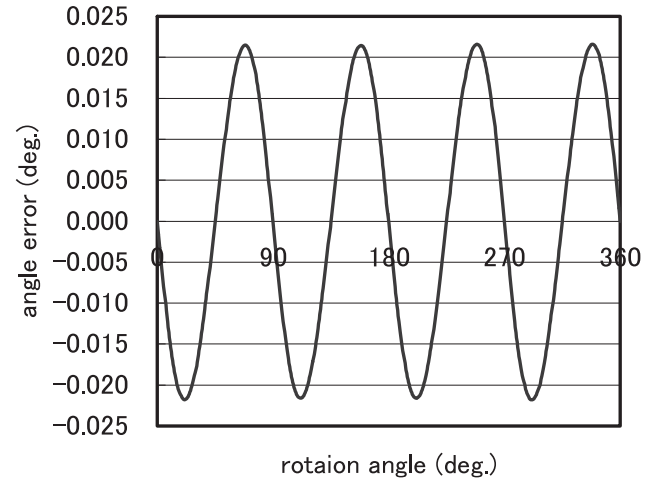

Fig. 11. Error in the computed angle as a function of the rotational encoder position the Hall effect device positions are then calculated from the flux distributions, and the output waveforms $\mathrm{Va}$, and $\mathrm{Vb}$ are obtained. Next, the characteristics of the encoder, such as the amplitudes and the distortion of the waveforms, the angle errors and accuracy are estimated. For expeditious parameter variation studies, the auto mesh generator was developed. The process sequence is thus automatically carried out.

4.3 Optimal Design To obtain an encoder with high accuracy, the parameter studies of the shape and material of an encoder head were carried out using FEM analysis. As show in Fig. 8, it is clear that the magnetic saturation of the stationary body increases the distortion of the waveform and results in the degradation of the computed angle accuracy.

The flux distribution of the encoder that was chosen finally is shown in Fig. 9. The output waveforms $\mathrm{Ba}$ and $\mathrm{Bb}$ are shown in Fig. 10. The distortion of the waveform is seen to be quite low and is about $0.03 \%$ of the fundamental wave. The error in the computed angle is only 0.04 degrees as shown in Fig. 11. The accuracy of the chosen design was determined to be $0.01 \%$, which corresponds to 13-bit accuracy.

\section{Test Results and Discussions}

The photograph of an unassembled rotary magnetic encoder is shown in Fig. 12. The magnet is attached to the shaft of the motor and the molded part that has the Hall effect devices in it is fixed to the stationary body. Tests were conducted on a prototype encoder using the test equipment shown in Fig. 13. The encoder was connected to a highly accurate reference encoder that had a resolution of $10^{6}$ pulses/rotation, for evaluating the accuracy. The accuracy was tested after adjusting the amplitudes, the phases and the offsets of the differential signals for phases A and B. The

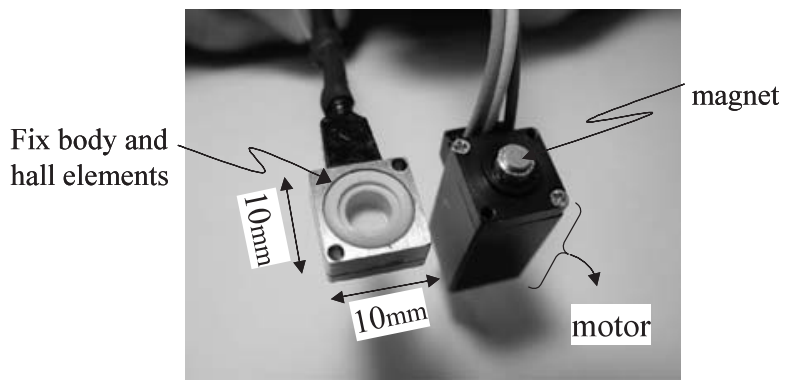

Fig. 12. Prototype of magnetic encoder

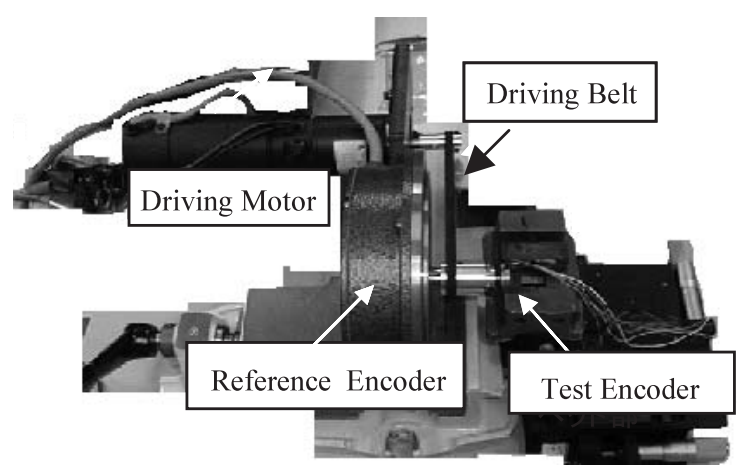

Fig. 13. Test setup 


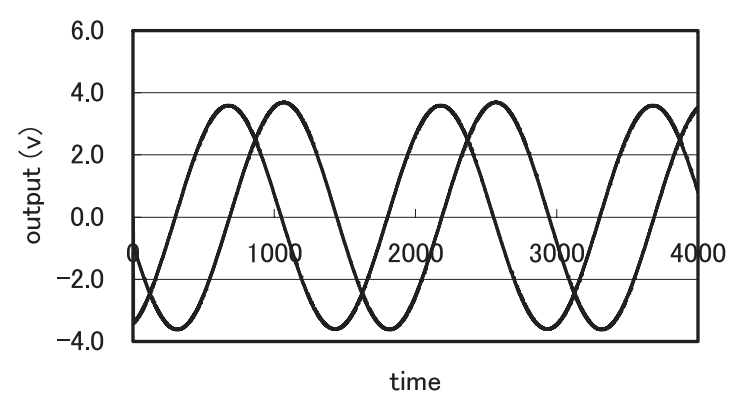

Fig. 14. Output signal of Phase A, B

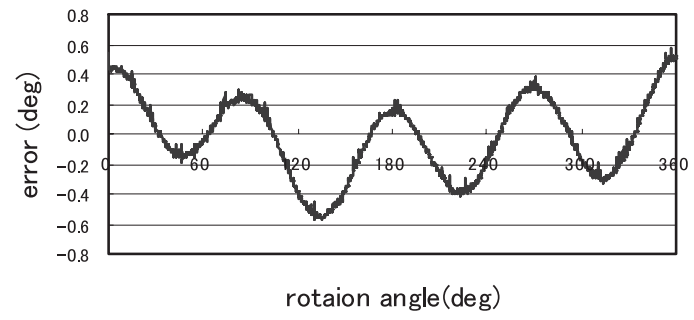

Fig. 15. Observed error in angle obtained from prototype encoder

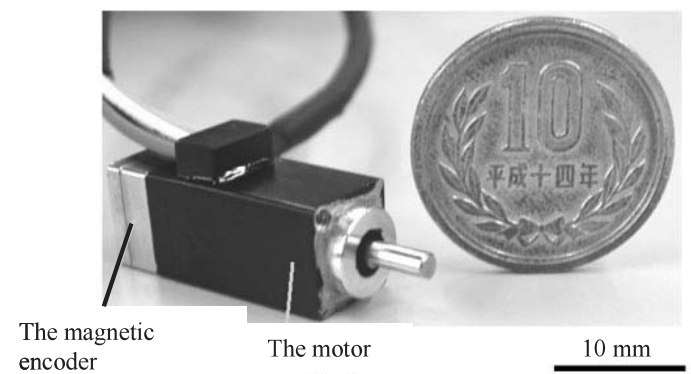

Fig. 16. Prototype Unit was tested

output waveforms of phases A, and B are shown in Fig. 14. The distortion obtained from these waveforms was $0.6 \%$ of the fundamental component. The distortions were greater than the simulation result. Fig. 15 shows the detection error based on the signals from the reference encoder. The angle error was 1.1 degrees. Therefore, the accuracy was $0.3 \%$ or 8 bit. The resolution of the prototype encoder was verified by giving a position command 10 revolutions to the servo amplifier. The resulting pulse count from the test encoder was 40960 pulses, which translated to $4096 \mathrm{ppr}$.

It is estimated that the differences of the results between the tests and the simulations was caused mainly by the non-linearity of the Hall effect devices, which has a maximum non-linearity of $2 \%$ according to the manufacturer's catalog. Another reason for the difference may also be due to the use of 2 dimensional FEM. The designing of the encoder head using 3D FEM is part of future work.

Based on simulation results (Fig. 11) and experimentally observed data (Fig. 15), the error in the computed angle is seen to follow a defenite sinusoidal pattern. In other words, the error is not random and this observation is important because it shows that the observed error should be fairly simple to compensate. Many methods are available to compensate for predictable error patterns. Use of such methods can help improve the overall accuracy of the proposed encoder.

\section{Conclusions}

A novel magnetic rotary encoder is proposed. The structure is very simple and the size is as small as $10 \mathrm{~mm}$. The structure and the operating theory were presented. And the optimal design of the encoder was carried out using the finite element method. The prototype was developed and evaluated using the high accuracy reference encoder. It was proved that the new type encoder has high accuracy of $0.3 \%$ ( 8 bit) and resolution of 4096 ppr. As shown in Fig. 16 the encoders have been applied to the servomotors used for small robots.

(Manuscript received March 10, 2005, revised Jan. 23, 2006)

\section{References}

( 1 ) H. Nakamura, T. Matsuo, K. Matsuzaki, K. Uemura, and T. Kabashima: "Development of Micro Servo Actuators", Proc. of 2nd International Workshop on Microfactories, pp.133-136 (2000)

( 2 ) Y. Kikuchi, F. Nakmura, H. Wakiwaka, H. Yamada, and Y. Yamamoto: "Consideration of Magnetization and Detection on Magnetic Rotary Encoder Using Finite Element Method", IEEE Trans. Magn., Vol.33, No.2, pp.21592162 (1997)

( 3 ) United States Patent US 6,762,897 BI: "Magnetic Encoder Apparatus", Jul.13 (2004)

( 4 ) European Patent EP 1014039AI: "Magnetic Encoder”, Nov.11 (2004)

Takefumi Kabashima (Member) was born in Fukuoka Prefecture

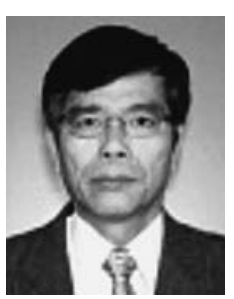

Engineers of Japan. in 1949. He received the B.S. degree in physics from Science Faculty of Kyusyu University, Fukuoka, Japan in 1972, and Ph.D. degree in Electrical Engineering from Okayama University, Japan in 2002. From 1972 he has developed the magnetic field analysis system using FEM and applied it to develop servomotors. His present interests include the development of new type servo sensors for servomotors. Dr. Kabashima is a member of the Institute of Electrical

Yuji Arinaga (Non-member) was born in Japan. He received the

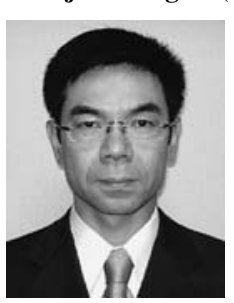
M.S. degree in electrical engineering from Kyushu Institute of Technology in 1989. In 1989, he joined Yaskawa Electric Corporation, where he is currently an assistant manager of the Mechatronics group in the R\&D Center. His present interests include optical sensors, magnetic sensors, and other sensor issues. Mr. Arinaga is a member of The Japan Society for Precision Engineering.

Koji Uemura (Non-member) was born in Japan. He received the B.S

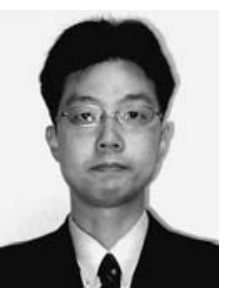
degree in physics from Tokyo University of Science in 1989. In 1989, he joined Yaskawa Electric Corporation, where he is currently an assistant manager of the Encoder Section. His present interests include magnetic materials, magnetic sensors, micro electro mechanical systems, and other sensor issues. Mr. Uemura is a member of The Japan Society of Applied Physics, and The Magnetics Society of Japan. 
Ikuma Murokita (Non-member) was born in Japan. He received the B.S degree in Mechanical engineering from Kyushu University in 1987. In 1987, he joined Yaskawa Electric Corporation, where he is currently an assistant manager of the Mechatronics group in the R\&D Center. His present interests include magnetic sensors and control software.
Motomichi Ohto (Member) was born in Oita prefecture, Japan, in 1961. He received the M.S., and Ph.D. degrees in electrical engineering from Oita University, Oita City, Japan, in 1985, and 2005, respectively. In 1986, he joined Yaskawa Electric Corporation, Kitakyushu, Japan, where he is a Manager of Mechatronics group in the R\&D Center. He is currently engaged in analysis of magnetic fields, and development of new servo motor products. Dr. Ohto is a member of IEE Japan. 